\title{
Grupo etario de 9-15 años periodo crítico para adquirir la adicción al tabaquismo y momento ideal para programas educativos de prevención.
}

\section{Age group of 9-15 years critical period to acquire the addiction to smoking and ideal time for prevention education programs.}

\author{
Enrique Manuel Donado Badillo. \\ Docente Universidad del Norte, Docente Universidad Libre, Escuela Superior de Administración Pública, Universidad Autónoma del Caribe. \\ Magíster en salud pública Universidad Nacional de Colombia., Doctorando en Administración de Sistemas \\ y Servicios de salud Universidad Olmeca México Especialista en Planificación y Administración de desarrollo Regional \\ de la Universidad de los Andes Bogotá Especialista en Finanzas públicas, Colombia, edonadob@hotmail.com \\ Moyra Striedinger de González \\ Magister en Administración de Empresas. Universidad del Norte y doctorando en Sistemas y Servicios de Salud. Universidad OLMECA, México. \\ Asesora Sistemas de Calidad en Unidad de Oncología Médica ONCOMEDIC, Barranquilla y Coordinadora Maestría en Calidad \\ en Servicios de Salud de la Universidad Autónoma del Caribe
}

Para citar este artículo:

Donado, B. E.; Striedinger, M. (2014). Grupo etario de 9-15 años periodo crítico para adquirir la adicción al tabaquismo y momento ideal para programas educativos de prevención. Escenarios. 12(1), 74-84.

\begin{abstract}
RESUMEN
En algunos países latinoamericanos como México se observa un marcado descenso en la edad de inicio del consumo de tabaco entre las generaciones nacidas en los años 40 , quienes empezaron a fumar pasados los veinte años, y la generaciones de la década de los 80 que empezaron el consumo en promedio a los trece años. Este estudio transversal determinó cuál es el grupo etario crítico para continuar con el hábito de fumar y adquirir la adicción y contrastarlo con otras edades de inicio que hacen posible que ellos mismos en edades futuras abandonen el hábito de fumar o sean más sensibles a programas de ayuda. Lo anterior requeriría realizar oportunamente, a ese rango de edad crítico, programas educativos preventivos. Para ello, se enfocó en la caracterización de una población estudiantil, como la Universidad Autónoma del Caribe que determinó una afectación del 11,41\% de la población estudiantil, dos veces más a los hombres que a las mujeres con diferencias estadísticamente significativas. La encuesta fue aplicada a una muestra aleatoria fue 377 con un instrumento validado por la Organización Mundial de la Salud (OMS) y se realizó un piloto, para su validez de contexto y semántica. En la encuesta se encontró que los que se iniciaron en el tabaquismo después de los 15 años, abandonaron el hábito un porcentaje mayor de ellos y que cuando se inicia fumando, en un rango de edad de 9 a 15 años, hace que más adelante sea una adicción, lo cual dificultaría los posteriores programas educativos para dejar el hábito.
\end{abstract}

Palabras Clave: Programas educativos de prevención, adicción a tabaquismo, grupo etario crítico.

\begin{abstract}
In some Latin American countries such as Mexico, there is a marked decrease in the age of onset of tobacco use among generations born in the 1940s, who started smoking after age twenty one, and the generation of the 1980s that started the consumption on average at age thirteen years This study determined what is the critical age group to continue with the habit of smoking and acquire addiction and compare it with other ages of home that make it possible for themselves in future ages to quit smoking or are more susceptible to aid programs. This would require timely, perform at that critical age range, preventive educational programmes. To do this, it focused on the characterization of a student population, as the Autonomous University of the affected Caribbean $11.41 \%$ of the student population, twice more to men than to women with statistically significant differences. The survey was applied to a random sample was 377 with instruments validated by the World Health Organization (who) and was a pilot for its validity on context and semantics. The survey found that which began in smoking after 15 years, abandoned the habit one higher percentage of them and that when it starts smoking, in a range from 9 to 15 years old, it makes later an addiction, which would make difficult the subsequent education Programs to quit.
\end{abstract}

Keys Words: Prevention education programs, smoking addiction, critical age group. 


\section{INTRODUCCIÓN}

La enfermedad denominada tabaquismo es un importante problema de salud Pública entre los estudiantes universitarios y al estudiar el tema en el campus de la Universidad Autónoma del Caribe se encontró que la problemática afecta al 11,41\% de la población estudiantil, dos veces más a los hombres que a las mujeres y en la misma proporción a quienes estudian en jornada diurna con relación a la nocturna con diferencias estadísticamente significativas Estudios realizados en México señalan que los años de educación media superior son un periodo crítico para el inicio de consumo de tabaco, más aún entre los estudiantes de mejor situación económica (Valdés-Salgado 2002).

Los actuales resultados empíricos son similares a los encontrados en otros estudios mundiales similares al mencionado. Todo ha conducido a establecer, mediante una investigación de corte transversal, el grupo etario sensible a adquirir el hábito de fumar, que resultó esta entre los 9 y 15 años, con una adicción mayor que el grupo etario pasados los 18 años de edad. Además el hábito de fumar crea una probabilidad mayor de consumir una segunda droga constituyéndose en la puerta de entrada al consumo de sustancias psicoactiva. De allí que el grupo de niños y adolescentes son especialmente vulnerables para el inicio del hábito (Tapia-Conyer 2001). Si se reconoce esta vulnerabilidad y se hace conciencia de ello, es esta la población de grupo etario objetivo donde deben actuar a nivel educativo, familia y sociedad en general, con importancia de diseñar e implementar programa y la necesidad de que las campañas educativos y de sensibilización, requieren que se realicen en el sistema educativo con el grupo etario resultante de la investigación.

\section{DESCRIPCIÓN DEL PROBLEMA}

La OMS publicó en mayo de 2013 que en el mundo hay mil millones de fumadores y que el $80 \%$ de los mismos habitan en países de ingresos medios o bajos. (OMS 2013). Estudios científicos han demostrado de manera inequívoca que el humo del cigarrillo es causa enfermedades, muertes y Discapacidad. El tabaco mata aproximadamente a la mitad de quienes lo consumen cifra que alcanza a seis millones de personas al año. De ellos más de cinco millones son consumidores o han consumido tabaco y más de 600.000 han estado expuestos al humo de tabaco ambiental o humo ajeno (OMS 2013). Los fumadores pasivos incluyen a embarazadas y a sus fetos, neonatos, lactantes, adolescentes y otros adultos en quienes tiene nefastos efectos (California Environmental Protection Agency 2005). El consumo de tabaco es una epidemia creciente en el mundo, sin fronteras, en el planeta una de cada diez defunciones son causadas por el consumo de tabaco; es decir, cada seis segundos muere una persona en la tierra a causa del tabaco. (OMS 2013, OMS 2008).que afecta a individuos de todas las religiones, sexos, razas, edades, estratos sociales debido principalmente a tres factores: Primero la nicotina contenida en el tabaco es una sustancia adictiva que luego de ser inhalada rápidamente llega al cerebro y genera cambios en el comportamiento, el efecto dura solo unos minutos, luego de ello el individuo experimenta síntomas de abstinencia que cesan si el paciente sigue fumando. El segundo es la mercadotecnia dirigida por la industria tabacalera a la población, aunada a su crecimiento demográfico y el tercero es su relativo bajo precio que es asequible a sus víctimas. (Mathers CD, Loncar D 2000).

Si persisten las tendencias actuales en el año 2030 el tabaco matará a ocho millones de personas por año y el $80 \%$ o más de estas muertes prematuras ocurrirán en países con ingresos medios o bajos. (OMS 2009). El tabaquismo causó cien millones de muertes en el siglo XX. Si se mantiene la tendencia actual, en el siglo XXI se registrarán hasta mil millones de muertes. (OMS 2013).

Los consumidores de tabaco que mueren prematuramente privan a sus familias de ingresos, aumentan el costo de la atención en salud y dificultan el desarrollo económico lo que constituye un enorme problema para sociedad en nuestros días (OMS 2013).

En Junio de 2013 La Sociedad Americana de cáncer y la Fundación mundial del Pulmón señalaron, en una publicación, que en América Latina existen 145 millones de fumadores y que el país que posee la prevalencia más alta de tabaquismo en la región es Chile. La prevalencia de tabaquismo en este país entre las mujeres es de $37 \%$ y entre los hombres alcanza el $44 \%$.( Sociedad Americana de cáncer y la Fundación mundial del Pulmón) En la costa Norte de Colombia se dispone de escasos estudios que permitan mostrar la magnitud del problema. En Barranquilla entre el segundo semestre de 2006 y el primer semestre de 2007 Luz Marina Alonso Pinedo determina los comportamientos de riesgo para la salud en estudiantes colombianos a su ingreso a una Universidad Privada de Barranquilla hallando una prevalencia de $35,2 \%$ superior a la encontrada en estudios realizados en Bogotá y el Magdalena. Entre los afectados el 25\% de ellos había intentado eliminar el hábito en el último año.

En las Universidades de Barranquilla, incluida la Universidad Autónoma del caribe es común hoy por hoy observar "colillas" y cajetillas vacías de cigarrillos en pasillos, escaleras, jardines, cafetería al igual que observar a estudiantes, profesores y funcionarios fumando en la institución lo que pone de relieve la presencia de una problemática que es necesario dimensionar, caracterizar, analizar y enfrentar. Todo en el contexto de marco jurídico, como la ley 1335 de 2009, del 21 de julio de 2009 expedida en Colombia, la cual en su capítulo $V$ establece la política para garantizar a los no fumadores espacios libres de humo de tabaco. En su artículo 19 prohíbe el consumo de tabaco y sus derivados en las instituciones de educación formal y no formal en todos sus niveles.

\section{METODOLOGIA}

El presente estudio de corte transversal fue realizado en las instalaciones de la Universidad Autónoma del Caribe de 
Barranquilla con el fin de determinar las características del hábito del tabaquismo y su prevalencia, entre los estudiantes de la Universidad Autónoma del Caribe de la Ciudad de Barranquilla, para posteriores campañas y programas educativos de prevención Entre los posibles factores de riesgo indagados se encuentran los siguientes: Relación de tabaquismo con: sexo, Jornada, estrato o nivel socioeconómico, causas que motivaron a su consumo inicial o hábito en diferentes grupos etarios y otros.

Fueron encuestados de manera anónima a través del aula virtual de la Universidad Autónoma del caribe un total de 377 estudiantes universitarios de la sede de Barranquilla con edades comprendidas entre 16 y 55 años que hicieron parte de una muestra estratificada por sexo, jornadas y niveles socioeconómicos obtenida de la base de datos de estudiantes matriculados en todas las facultades y programas de pregrado y posgrado de la Universidad Autónoma del caribe a mediados de 2013, la cual luego de depurada contó con una población de 4.808 mujeres y 6085 hombre para un total de 10893 estudiantes.

El instrumento aplicado fue el de Tabaquismo y Juventud de la Organización Mundial de la Salud que cuenta con 69 preguntas algunas abiertas otras cerradas que permiten conocer cinco características importantes del fenómeno de tabaquismo: Consumo de tabaco directamente realizado por la persona, Exposición al humo producido por otras personas que fuman, Actitud acerca de dejar de fumar cigarrillos, Orientaciones recibidas en la Universidad acerca del tabaco y consumo de tabaco y otras sustancias. Como Objetivo se determinó las características socio humanas de los estudiantes en relación con el tabaquismo en la Universidad Autónoma del Caribe, para elaboración de campañas y programa de prevención, sensibilización y abandono del hábito.

\section{POSTURA TEÓRICA}

En nuestros días, el consumo de tabaco, se realiza más frecuentemente mediante la inhalación del humo producto de la combustión de los productos del tabaco. El cigarrillo en el extremo encendido durante las bocanadas alcanza temperaturas entre bocanadas de $400^{\circ} \mathrm{C}$, y la combustión transforma muchos componentes originales de la planta del tabaco debido a complejas reacciones químicas las cuales pueden estar presentes en el proceso de fumar. Según la Sociedad Americana de cáncer se han identificado más de 7000 sustancias químicas en el humo de tabaco en sus estados, gaseoso y de partículas.(American Cáncer Society 2012)

El humo de tabaco Ambiental (HTA) es la combinación de los dos anteriores también denominado de segunda mano y es el humo que está en suspensión en un ambiente donde hay personas fumando. El $85 \%$ proviene de la corriente lateral del humo de cigarrillo en combustión y el otro $15 \%$ corresponde al humo de la corriente principal espirado por los fumadores activos (Sergio Bello 2005).
Recientemente en una publicación del programa del control de tabaco del ministerio de salud de Argentina aparece descrito un humo de tercera mano que es aquel que "describe la mezcla invisible de gases y partículas que incluyen metales pesados, material radioactivo y sustancias cancerígenas que se adhieren al pelo, ropa de los fumadores, muebles, cortinas, alfombras o tapizados y que permanecen o se desprenden luego de que el fumador ha apagado el cigarrillo" estas sustancias pueden permanecer en la habitación o sitio donde se estuvo fumando hasta dos semanas después de haber sido ventilada. Se sabe que más de 250 de estos químicos son perjudiciales, y al menos 69 causan cáncer.

Es necesario diferenciar tres conceptos en relación a los componentes del tabaco: Ingredientes del tabaco son todos los componentes del producto, que incluyen los materiales utilizados para fabricar dichos componentes sumados a las sustancias residuales de las prácticas agrícolas, del procesamiento, del almacenamiento $\mathrm{y}$ aquellas sustancias que pueden pasar del envase al producto.

Emisiones de los productos del tabaco comprenden las sustancias que realmente se ponen en contacto con el fumador. Las emisiones son sustancias generadas al utilizar el producto.

Exposición, término que se refiere a la fracción de las emisiones que absorbe efectivamente el fumador y son la causa de la adicción, enfermedades, discapacidad y muertes atribuibles al tabaco.

Las emisiones son inhaladas directamente por quien fuma, a este individuo se le denomina simplemente fumador o fumador activo y también por aquellas personas de no tienen el hábito de fumar, pero están en contacto o expuestas a las emisiones de los productos del tabaco (humo ambiental) aéstas las denominamos fumadores involuntarios o pasivos.

Los fumadores pasivos incluyen a los fetos de mujeres embarazadas que fuman de manera activa o pasiva ya que algunos productos del tabaco como la nicotina y el monóxido de carbono atraviesan labarrera placentariayocasionan alteraciones fetales.

\section{Epidemiologia del Tabaquismo}

Estudiando y analizando el comportamiento del consumo de tabaco en diferentes países se puede establecer que el tabaquismo tiene un patrón propio en cada país, pero la observación de la evolución a nivel internacional ha hecho posible el registro y elaboración de algunos patrones que pueden ser útiles para establecer pronósticos y tomar decisiones. Desde una perspectiva epidemiológica se han podido identificar en esta evolución del tabaquismo, cuatro fases que caracterizan la epidemia tabáquica.

La fase I se caracteriza por registrar un consumo bajo en los hombres (prevalencia inferior al $15 \%$ en los varones), y muy inferior o inexistente entre las mujeres ( $<5 \%)$. El consumo promedio de cada adulto es inferior a 500 cigarrillos por año, por ello la morbimortalidad asociada al consumo de tabaco es poco evidente. En esta 
fase los datos de mortalidad no permiten apreciar las muertes atribuibles al tabaco. La duración de esta etapa oscila entre 10 y 20 años. En los países donde se ha estudiado, en esta fase el fumar era una práctica socialmente aceptada, sin restricciones para el consumo por existir otras prioridades de salud pública.

La fase II tiene las siguientes características: La prevalencia de tabaquismo aumenta rápidamente en varones y lentamente entre las mujeres alcanzando niveles del 50 - $80 \%$ en los primeros, los exfumadores son muy escasos. El hábito de fumar está extendido a todas las clases sociales, durante esta fase la prevalencia del hábito en las mujeres es menor con relación a la de los hombres, pero es creciente ya que presenta un retraso de una a dos décadas con relación a los hombres. El consumo promedio anual por adulto alcanza entre 1000 a 3000 cigarrillos y entre los varones oscila entre 2000 y 4000 cigarrillos consumidos al año. Al finalizar esta fase un $10 \%$ de las muertes en varones son atribuibles al tabaco. Esta fase suele durar entre dos a tres décadas y en la mayoría de los países durante esta fase se realizan las primeras acciones de prevención.

En la fase III hay un descenso de la prevalencia en varones hasta el $40 \%$ aproximadamente, los exfumadores son un segmento considerable y creciente entre los varones al tiempo que ocurre una larga estabilización del hábito entre las mujeres sin alcanzar los niveles de prevalencia que hubo en la población masculina. El consumo promedio anual por adulto es el mayor en esta fase alcanzando cifras de 3000 a 4000 cigarrillos consumidos por año. La mortalidad asociada al consumo de tabaco es de $25-30 \%$ del total de muertes entre los varones, y $5 \%$ en las mujeres. Durante esta fase los programas de control de tabaquismo están más integrados y coordinados y son realizadas las primeras campañas de prevención en las escuelas. La duración de esta fase es de tres décadas.

La fase IV tiene las siguientes características: La prevalencia disminuye en ambos sexos, entre hombres y mujeres tiende a ser la misma ya que en la población masculina baja a un $35 \%$ mientras que en la femenina llega al 30\%. La mortalidad atribuible al tabaco alcanza el $35 \%$ en hombres y 25 en las mujeres y empieza a descender progresivamente. En esta fase los segmentos de población de mayor nivel económico y de instrucción han abandonado masivamente el hábito.

Con el transcurrir del tiempo es posible que aparezca una quinta fase caracterizada por la notoria disminución de la mortalidad atribuible al tabaquismo tanto en hombres como en mujeres. (López 1994) (Villabi 2000)

\section{Epidemiologia del Tabaquismo en el Adolescente.}

Sánchez afirma que tanto el inicio del consumo de tabaco como el mantenimiento del mismo, como ocurre con otras drogodependencias, tienen su origen en el entorno social del niño o adolescente y se consolida debido a otras características biológicas o psicológicas del individuo. (Sánchez 2004)1. Entre los factores sociales que más influyen en el inicio de consumo de tabaco se pueden citar: fracaso escolar, manifestaciones de inadaptación social y escolar (González 1999)2. Entre los posibles factores predictores al consumo se mencionan: existencia de amigos fumadores, actitudes favorables al consumo, intuición de fumar en el futuro, consumo de bebidas alcohólicas (Ariza 2002)3 En los países desarrollados, el tabaco suele ser la primera sustancia adictiva con la que se ponen en contacto la mayoría de adolescentes y escolares y el $90 \%$ de los fumadores inician su hábito en esta etapa de la vida. (47) (Jiménez 2004).

Como parte de un proceso de experimentación y conocimiento o exploración del entorno propio del adulto, el niño o adolescente comienza a fumar. Hay autores que señalan el consumo de tabaco en adolescentes como una forma de afrontar el estrés en este complejo período del ciclo vital, así el consumo de tabaco es utilizado como regulador emocional y desarrolla la dependencia a esta sustancia (Waldran 1990-Tomeo 1999).

\section{Grupo Etario De Inicio y Escolaridad}

La OMS afirma que en el ámbito mundial se ha descrito un descenso en la edad de inicio de fumar (OMS 1999), se estima que diariamente empiezan a fumar entre 68 mil y 84 mil niños en los países de bajos y medianos ingresos (OPS 2000) (pág 127). En algunos países latinoamericanos como México se observa un marcado descenso en la edad de inicio del consumo de tabaco entre las generaciones nacidas en los años 40 quienes empezaron a fumar pasados los 21 años y la Generaciones de la década de los 80 que empezaron el consumo a los 13 años (Secretaria de Salud de México) Algunos estudios realizados en México señalan que los años de educación media superior son un periodo crítico para el inicio de consumo de tabaco, más aún entre los estudiantes de mejor situación económica (Valdes-Salgado 2002).

En estudios realizados en Estados Unidos informan que la prevalencia de tabaquismo aumenta con la edad a lo largo de la adolescencia. En estos estudios se considera fumador a aquel que ha consumido tabaco en el último mes (CDC 2008b) En un estudio denominado Monitoring the Future ( Johnston et al. 2007 ) muestran que el tabaquismo actual se informó en un 8,7 por ciento de los estudiantes de octavo grado, el 14,5 por ciento de los alumnos del grado 10 , y el 21,0 por ciento de los estudiantes del grado 12 . En el mismo estudio se encontró que los adolescentes que más fuman son aquellos que tienen padres con menos educación, menos aspiraciones para la educación superior, y la residencia rural. En este país la frecuencia y cantidad también aumenta, es así como en el estudio se encontró que el tabaquismo diario se informó en un 4,0 por ciento de los estudiantes de octavo grado, el 7,6 por ciento de los alumnos del grado 10 , y el 12,2 por ciento de los estudiantes del grado 12. En relación a la cantidad de cigarrillos consumidos el comportamiento fue así: 1.5 por ciento de los estudiantes de octavo grado , el 3,3 por ciento de los alumnos del grado 10 , y el 5,9 por ciento de los estudiantes del grado 12 fuman la mitad de un paquete o más cigarrillos al día ( Johnston et al. 2007). 


\section{Efectos Del Consumo De Tabaco Sobre La Salud.}

Los efectos del consumo de tabaco son: Biológicos, Económicos, sociales y ambientales Los efectos Biológicos del cigarrillo involucran: Adicción, Enfermedad, Discapacidad y muerte. En cuanto a la adición:

Adicción: El Ministerio de Salud de Los Estados Unidos en 1988 clasificó a la nicotina, componente del cigarrillo, como una sustancia adictiva. La nicotina es adictiva en los seres humanos debido a que una porción de la molécula de la nicotina es similar a la acetilcolina, un neurotransmisor cerebral importante (Brody et al. 2006) El tabaco genera dependencia con un mecanismo similar a la cocaína o a la heroína. Los cigarrillos proveen al organismo nicotina 1 a $2 \mathrm{mg}$ por unidad consumida. Luego de inhalar el humo del tabaco, la nicotina contenida en el mismo llega al cerebro en 10 segundos lo que contribuye a su poder de adicción.

La nicotina causa dos veces más adicción que la heroína, cuatro veces más que la cocaína y más de siete veces que la marihuana como se puede apreciar en el cuadro que se presenta a continuación:

Cuadro 1. Porcentaje de usuarios que se torna dependiente con relación a la edad media del primer uso del cigarrillo

\begin{tabular}{|l|c|c|c|c|}
\hline \multicolumn{1}{|c|}{ Sustancia } & Acceso & $\begin{array}{c}\text { Capacidad } \\
\text { de causar } \\
\text { dependencia** }\end{array}$ & Letalidad & $\begin{array}{c}\text { Precocidad } \\
* * *\end{array}$ \\
\hline $\begin{array}{l}\text { Nicotina } \\
\text { (del cigarrarillo) }\end{array}$ & Grande & 80 & Alta & 15,5 \\
\hline Heroína & Bajo & 35 & Media & 19,5 \\
\hline Cocaína & Medio & 22 & Alta & 21,9 \\
\hline Sedantes* & Medio & 13 & Media & 19,5 \\
\hline Estimulantes* & Medio & 12 & Alta & 19,3 \\
\hline Marihuana & Medio & 11 & Baja & 18,4 \\
\hline Alucinógenos & Grande & 9 & Baja & 18,6 \\
\hline Alcohol & Grande & 6 & Media & 17,4 \\
\hline Tranquilizantes* & Medio & 5 & Media & 21,2 \\
\hline Inhalantes & Grande & 3 & Media & 17,3 \\
\hline
\end{tabular}

* Uso no médico

** \% de usuarios que se torna dependiente

*** Edad media del primer uso, en años

Fuente: Investigación Nacional Domiciliaria sobre uso de drogas en Estados Unidos. National Health Institute, 2001.

Según lo publicado por el ministerio de salud de Argentina (2014) El consumo de tabaco genera tres formas de dependencia:
- Física: provocada por la acción directa de la nicotina sobre el sistema nervioso central, responsable del síndrome de abstinencia.

- Psicológica: el hábito de fumar se constituye en la compañía del hombre en diferentes situaciones y se torna difícil cambiar esta situación.

- Social: el fumar es aún un acto social, se hace en grupos, en reuniones con amigos, y entre los adolescentes en muchas ocasiones, adquiere un significado de rebeldía y de madurez.

Algunos estudios sugieren que los adolescentes reportan síntomas de dependencia, incluso a bajos niveles de consumo de cigarrillos (Colby et al 2000a, b;. Hughes 2001; DiFranza et al 2002b; Panday et al 2007). La diferencia en la sensibilidad a la nicotina en los adolescentes y los adultos también se reporta en modelos animales (Slotkin 2002;Adriani et al 2003; Torres et al 2008). Por ejemplo, Levin y sus colegas (2003) encontraron que cuando las ratas fueron expuestas primero a la nicotina en la adolescencia, se auto-administraron más nicotina aquellas ratas expuestas en la edad adulta.

Estas diferencias en la auto-administración por edad de la primera exposición persisten en la edad adulta. Del mismo modo, Beluzzi y sus colegas (2004) encontraron que una sola inyección de nicotina durante la adolescencia temprana fue suficiente para establecer la preferencia de lugar condicionado en ratas, mientras que tales inyecciones en la adolescencia tardía o la edad adulta no eran suficientes.

Las enfermedades crónicas causadas por el consumo de tabaco son responsables de las principales causas de muerte y discapacidad en muchos países del mundo e incluyen enfermedad Pulmonar obstructiva crónica, Cáncer de Pulmón, Bronquitis Crónica, Leucemias, Angina de pecho, infarto agudo de miocardio, trombosis cerebral, cáncer de vejiga, páncreas, hígado, riñón.

\section{PRESENTACIÓN Y ANÁLISIS DE RESULTADOS.}

Fueron encuestados de manera anónima a través del aula virtual de la Universidad Autónoma del caribe un total de 377 estudiantes universitarios de la sede de Barranquilla con edades comprendidas entre 16 y 55 años que hicieron parte de una muestra estratificada por sexo, jornadas y niveles socio económicos obtenida de la base de datos de estudiantes matriculados en todas las facultades y programas de pregrado y posgrado de la Universidad Autónoma del caribe durante el segundo semestre de 2013, la cual luego de depurada contó con una población de 4.808 mujeres y 6085 hombre para un total de 10893 matriculados. La encuesta realizada fue la de Tabaquismo y Juventud de la Organización Mundial de la Salud que cuenta con 67 preguntas algunas abiertas, otras cerradas que permiten conocer cinco características importantes del fenómeno de tabaquismo entre las que se encuentran: Exposición al humo producido por otras personas que fuman, Actitud acerca 
de dejar de fumar cigarrillos, Conocimientos de los mensajes de los medios de comunicación y publicidad acerca del fumar, Enseñanzas recibidas en la Universidad acerca del tabaco y consumo de tabaco y otras sustancias.

\section{MAGNITUD DEL PROBLEMA Y POBLACIÓN ESTUDIANTIL AFECTADA}

Los resultados muestran que el 59,68\%de los estudiantes de la universidad nunca han fumado (No Fumadores) y 40,32\% afirma haber probado el cigarrillo al menos una vez en su vida (Ex fumadores o fumadores). Este porcentaje es inferior al encontrado por la OMS en Bogotá en el año 2002 al aplicar la misma encuesta en adolescentes que arrojó un resultado de $62 \%$ de estudiantes que habían probado el cigarrillo al menos una vez en su vida. (ver cuadro 2).

Cuadro 2. Prevalencia de estudiantes que han probado el cigarrillo al menos una vez en la vida

\begin{tabular}{|c|c|c|c|}
\hline $\begin{array}{c}\text { Han probado } \\
\text { el cigarrillo }\end{array}$ & $\mathrm{Si}$ & No & Total \\
\hline Estudiantes & 152 & 225 & 377 \\
\hline Proporción & 0,40318302 & 0,59681698 & 1 \\
\hline \% & 40,3183024 & 59,6816976 & 100 \\
\hline intervalo de & $0.35232946-$ & $0.5459634-$ & \\
confianza 95\% & 0.45403656 & 0.6476705 & \\
\hline
\end{tabular}

Fuente: Los autores
Gráfico 1. Porcentaje de estudiantes que han probado el cigarrillo al menos una vez en su vida

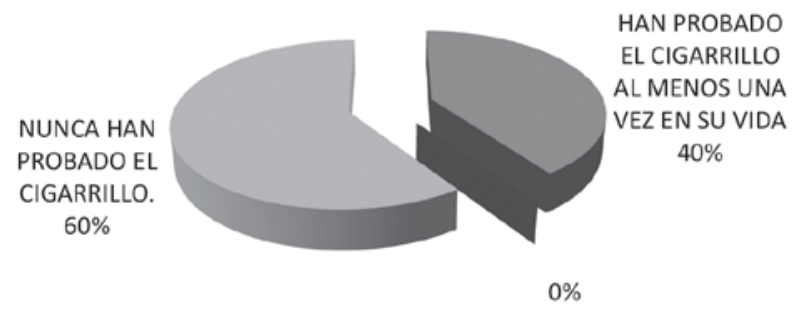

Fuente: Los autores

La encuesta determinó cuántos de aquellos que probaron el cigarrillo persisten en fumar al menos una vez dentro del último mes y a estos los denominamos fumadores. El resultado se muestra en la gráfica $\mathrm{N}^{\circ} 1$ y su análisis posterior (Ver cuadro 3.) permitió determinar, que por ejemplo del 40,32\% que probaron al menos una vez en su vida el cigarrillo el $71,71 \%$ de ellos no adquieren el hábito de fumar o lo abandonan y solo el 28,91\% de los mismos afirmaron fumar durante el último mes.

Los resultados enseñan que la enfermedad denominada tabaquismo es un importante problema de salud Pública entre los estudiantes de la Universidad Autónoma del caribe que afecta al 11,41\% (IC: 8,1-14,7\%) de la población estudiantil (fumadores). Se estima de esta forma que la población afectada en la Universidad oscila entre 878 y 1607 estudiantes con un 95\% de probabilidad. De conformidad con la prevalencia 1.243 estudiantes están afectados. w

Cuadro 3. Característica de los estudiantes de la AUC según su exposición al cigarrillo durante el segundo semestre de 2013

\begin{tabular}{|l|c|c|c|c|}
\hline Característica & $\begin{array}{c}\text { Proporción de estudiantes } \\
\text { con relación al cuadro anterior }\end{array}$ & $\begin{array}{c}\text { Proporción actual } \\
\mathbf{2 0 1 3}\end{array}$ & $\begin{array}{c}\mathbf{\%} \text { actual } \\
\mathbf{2 0 1 3}\end{array}$ & $\begin{array}{c}\text { Intervalo de confianza } \\
\mathbf{9 5 \%}\end{array}$ \\
\hline Ex fumadores & $109 / 152=71.71 \%$ & 0,28912467 & 28,91246684 & $(24,2025-33,62241)$ \\
\hline Fumadores & $43 / 152=28.91 \%$ & 0,11405836 & 11,40583554 & $(8,063697-14,747973)$ \\
\hline no han fumado & 225 & 0,59681698 & 59,68169761 & $0.5459634-0.6476705$ \\
\hline Total & 377 & 1 & 100 & \\
\hline
\end{tabular}

Fuente: Los autores

Cuadro 4. Prevalencia del hábito de fumar entre los estudiantes de la Universidad Autónoma del Caribe durante el segundo semestre de 2013

\begin{tabular}{|l|c|c|c|c|}
\hline \multicolumn{1}{|c|}{ Fuman } & Estudiantes & Proporción & \% & Intervalo de confianza 95\% \\
\hline $\mathrm{Si}$ & 43 & 0,11405836 & 11,40583554 & $(8,063697-14,7479)$ \\
\hline No & 334 & 0,88594164 & 88,59416446 & $(85,25202-91,9363)$ \\
\hline Total & 377 & 1 & 100 & \\
\hline
\end{tabular}

Fuente: Los autores 
Gráfico 3. Prevalencia de tabaquismo entre los estudiantes de la Universidad Autónoma del Caribe durante el segundo semestre de 2013

\section{- HAN FUMADO DURANTE EL ÚLTIMO MES - NO HAN FUMADO DURANTE EL ÚLTIMO MES}

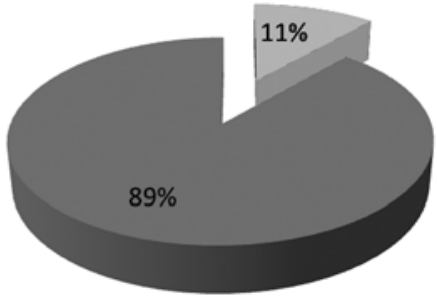

Fuente: Los autores

También se estableció la relación del hábito de fumar con el sexo entre los estudiantes. Los resultados muestran que 12 de 162 mujeres encuestadas fuman (han fumado durante el último mes) y lo hacen 31 de 215 hombres encuestados. Así la prevalencia del hábito de fumar entre las mujeres es de 7,40\% (IC: 3,07 -11,74\%) entre los hombres la misma prevalencia es de 14,42\% (IC: 9,4919,35\%) la razón hombre mujer es cercana a 2:1. Como la muestra fue estratificada por sexo, se determinó si las diferencias eran debido al azar o no y se encontró que el OR es de 2,11 (IC: 1,001$4,15)$ p: 0,0034 lo que nos permite afirmar que la prevalencia de tabaquismo entre los hombres es el doble que entre las mujeres.

El proceso estadístico se construyó con software SPSS a un intervalo e confianza del 95\% Ver Cuadro 5.

Cuadro 5. Diferencia en prevalencia entre hombres y mujeres en los estudiantes de la UAC durante el segundo semestre de 2013

\begin{tabular}{|l|c|c|c|}
\hline Sexo & Hombre & Mujeres & Total \\
\hline Fuman & 31 & 12 & 43 \\
\hline No fuman & 184 & 150 & 334 \\
\hline Total & 215 & 162 & 377 \\
\hline Prevalencia & $14,42 \%$ & $7,41 \%$ & $11,41 \%$ \\
\hline OR & 2,1 & 0,47 & \\
\hline p & 0,034 & 0,034 & \\
\hline
\end{tabular}

Fuente: Los autores con software SPSS.

Por otro lado, la prevalencia de tabaquismo en las jornadas Diurna y nocturna y si había diferencias estadísticamente significativas. Los resultados permiten mostrar lo siguientes: 32 de los fumadores de 226 encuestados estudian en jornada diurna siendo la prevalencia de tabaquismo entre los que estudian en Jornada diurna es de 14,16\% (IC 0,0939-0,1892) mientras que en lajornada nocturna es de 7,28\% (IC: 2,81-0,12). Un OR 2,09 (1,0232-4,307) valor de p: 0,039. Este resultado también permite establecer que los estudiantes de la diurna fuman dos veces más que los de la Jornada Nocturna siendo las diferencias estadísticamente significativas. (Ver cuadro 6).

Cuadro 6. Diferencia en prevalencia entre los estudiantes de la jornada diurna con los de la jornada Nocturna en la UAC durante el segundo Semestre de 2013

\begin{tabular}{|l|c|c|c|}
\hline Jornada & Diurna & Nocturna & Total \\
\hline Fuman & 32 & 11 & 43 \\
\hline No fuman & 194 & 140 & 334 \\
\hline Total & 226 & 151 & 377 \\
\hline prevalencia & $14,16 \%$ & $7,28 \%$ & $11,41 \%$ \\
\hline IC 95\% & $9,391767-$ & $2,807554-$ & $(8,063697-$ \\
\hline OR & 18,926817 & 11,761982 & $14,7479)$ \\
\hline p & 2,09 & 0,47 & \\
\hline
\end{tabular}

Fuente: Los autores con software SPSS

Momento de inicio y causa del tabaquismo en la Universidad Autonoma del Caribe segundo semestre 2013.

A continuación (Cuadro 7) se analizó el grupo etario para contrastarlo con los estudios y teorías encontrados:

Cuadro 7. Edad de probar el cigarrillo entre los estudiantes de la Universidad Autónoma del Caribe desde antes de 10 años hasta 16 o más años. II Semestre de 2014

\begin{tabular}{|c|c|c|c|c|c|}
\hline 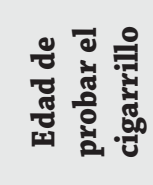 & 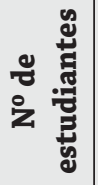 & 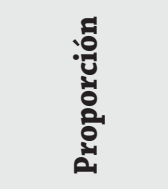 & $B^{\circ}$ & 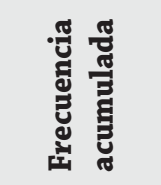 & $B^{\circ}$ \\
\hline $\begin{array}{l}10 \text { años o } \\
\text { menos: }\end{array}$ & 10 & 0,06578947 & 6,5789 & 0,0657895 & 6,5789 \\
\hline 11 años: & 2 & 0,01315789 & 1,3158 & 0,0789474 & 7,8947 \\
\hline 12 años: & 7 & 0,04605263 & 4,6053 & 0,1250000 & 12,500 \\
\hline 13 años: & 4 & 0,02631579 & 2,6316 & 0,1513158 & 15,132 \\
\hline 14 años: & 11 & 0,07236842 & 7,2368 & 0,2236842 & 22,368 \\
\hline 15 años: & 21 & 0,13815789 & 13,816 & 0,3618421 & 36,184 \\
\hline $\begin{array}{l}16 \text { años o } \\
\text { más: }\end{array}$ & 97 & 0,63815789 & 63,816 & 1 & 100 \\
\hline Total & 152 & 1 & 100 & & \\
\hline
\end{tabular}

Fuente: Los Autores

El inicio del consumo de Tabaco entre los estudiantes de la Universidad Autónoma del caribe ocurrió desde antes de cumplir 10 años (niñez) en un 6,6\% y durante la adolescencia. El periodo en que 
más se prueba el cigarrillo es de 16 años o más (63,82\%), tal como se aprecia en la tabla anterior y en la gráfica que a continuación se presenta. Esta edad coincide con el inicio de clases del adolescente en la Universidad. (Ver gráfico 4)

Gráfico 4. Edad de probar el cigarrillo entre los estudiantes de la UAC 2013

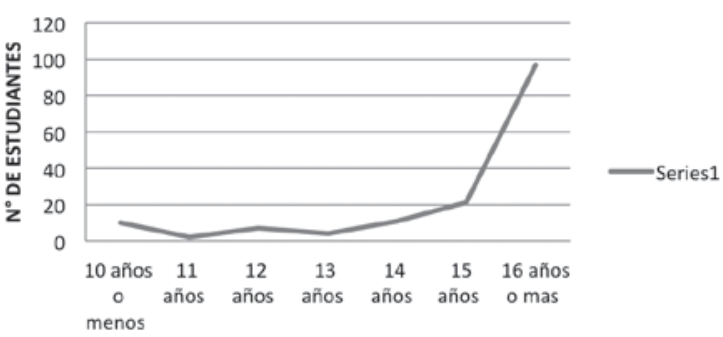

Fuente: Los autores

En la gráfica que se presenta a continuación se aprecia la cantidad que iniciaron el consumo de tabaco desde antes de los 10 años hasta más de 16 años y se aprecia que un 36,2 inició el consumo de tabaco antes de cumplir los 15 años de edad, el resto lo realizó después de esa edad. Con claridad (ver gráfico 5) se aprecia que el mayor consumo ocurre en los momentos en que la mayoría termina su bachillerato e ingresa a la Universidad (63,8\%).

Gráfico 5. Comparativo entre la frecuencia de estudiantes que iniciaron antes de los 15 años y después de esta edad en la UAC

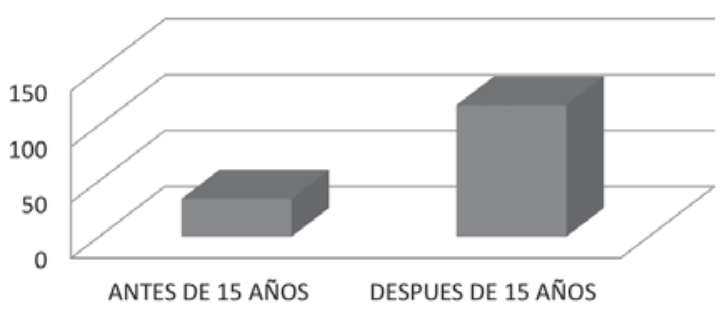

Fuente: Los autores

Al indagar la motivación que llevó a los estudiantes probar el tabaco o cigarrillo las respuestas obtenidas fueron las siguientes:

Gráfico 6. Motivación que llevó a los estudiantes a probar el cigarrillo.

$1 \%$

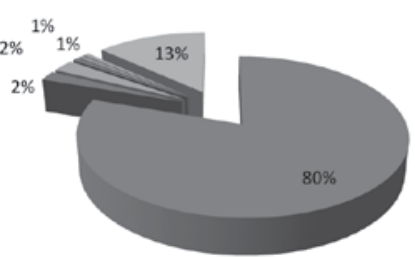

= CURIOSIDAD

- IMPRESIÓN DE LOS AMIGOS

- PARA SENTIRSE PARTE DE UN GRUPO

- PARA PARECER DE MAS EDAD

- PARA TENER MAS PERSONALIDAD

Fuente: Los autores

Se aprecia con facilidad que al $80 \%$ de los que probaron el cigarrillo los motivó la curiosidad y $13 \%$ otra causa no explorada lo cual ocurrió con mayor frecuencia después de los 15 años de edad. Al examinar las causas que motivaron a los que actualmente fuman lo encontrado fue lo siguiente. Es la curiosidad sin duda un factor de riesgo que motiva a los adolescentes a la incidencia de tabaquismo.

Al comparar las causas generales que motivaron a probar el cigarrillo o tabaco con las causas que motivaron el inicio del tabaquismo entre los estudiantes afectados lo encontrado es lo siguiente. (Ver gráfico 6)

Gráfico 7. Factor de la motivación de los actuales fumadores tabaquismo entre los los estudiantes de la UAC fumadores segundo semestre 2013.

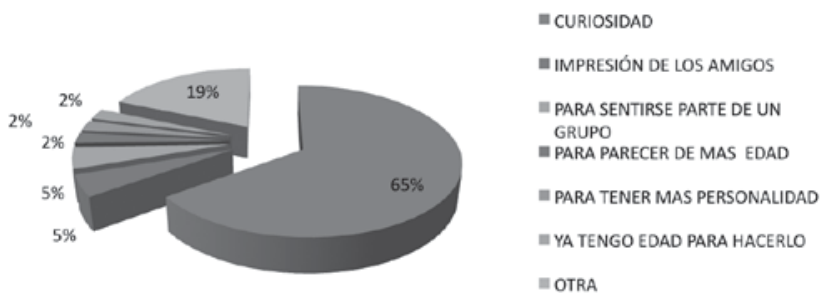

Fuente: Los autores

Entre los estudiantes que fuman el $65 \%$ lo hizo por curiosidad, pero en este grupo la participación de los amigos juega un papel más importante ya que un $10 \%$ lo hace bien sea para impresionar a los amigos o para sentirse parte de un grupo. Un 19\% de las causas son desconocidas y no fueron exploradas en la encuesta. $\mathrm{Al}$ analizar la curiosidad como factor de riesgo de tabaquismo se encontró que es estadísticamente significativo OR: 4,77(IC: 2,33-9,85)

\section{Quienes abandonan o no adquieren el hábito.}

Cuadro 8. Estudiantes que luego de probar el cigarrillo no adquieren o abandonan el hábito de fumar.

\begin{tabular}{|c|c|c|c|c|c|}
\hline $\begin{array}{l}\text { Edad de probar } \\
\text { el cigarrillo }\end{array}$ & 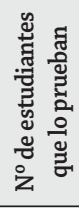 & 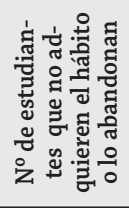 & 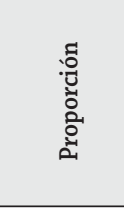 & 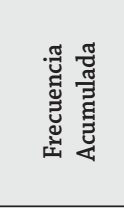 & $b^{\circ}$ \\
\hline 10 años o menos: & 10 & 5 & 0,04587 & 0,04587 & 4,58715 \\
\hline 11 años: & 2 & 0 & 0 & 0,04587 & 4,58715 \\
\hline 12 años: & 7 & 3 & 0,02752 & 0,07339 & 7,33944 \\
\hline 13 años: & 4 & 1 & 0,00917 & 0,08256 & 8,25688 \\
\hline 14 años: & 11 & 6 & 0,05504 & 0,13761 & 13,7614 \\
\hline 15 años: & 21 & 14 & 0,12844 & 0,26605 & 26,6055 \\
\hline 16 años o más: & 97 & 80 & 0,73394 & 1 & 100 \\
\hline Total & 152 & 109 & 1 & & \\
\hline
\end{tabular}

Fuente: Los Autores 
En el cuadro se aprecia como los que prueban el cigarrillo antes de los 16 años mantienen el hábito en un $73,4 \%$, solo lo abandona el 26,6\% en tanto que quienes lo prueban después de los 16 años el abandonan el hábito es de 73,4\% la diferencia de comportamiento entre uno y otro grupo es estadísticamente significativa.

\section{Quienes no abandonan el cigarrillo y adquieren el habito}

Cuadro 9. Edad en que los estudiantes fumadores prueban el cigarrillo y adquieren el hábito de fumar.

\begin{tabular}{|c|c|c|c|c|c|}
\hline 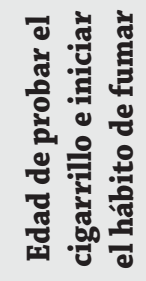 & 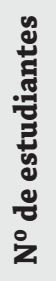 & 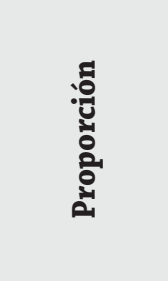 & $0^{\circ}$ & 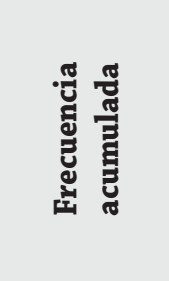 & $0^{\circ}$ \\
\hline $\begin{array}{l}10 \text { años } \\
\text { o menos: }\end{array}$ & 5 & 0,11627907 & 11,62791 & 0,11627907 & 11,6279 \\
\hline 11 años: & 2 & 0,04651163 & 4,651163 & 0,1627907 & 16,2791 \\
\hline 12 años: & 4 & 0,09302326 & 9,302326 & 0,25581395 & 25,5814 \\
\hline 13 años: & 3 & 0,06976744 & 6,976744 & 0,3255814 & 32,5581 \\
\hline 14 años: & 5 & 0,11627907 & 11,62791 & 0,44186047 & 44,186 \\
\hline 15 años: & 7 & 0,1627907 & 16,27907 & 0,60465116 & 60,4651 \\
\hline $\begin{array}{l}16 \text { años } \\
\text { o más: }\end{array}$ & 17 & 0,39534884 & 39,53488 & 1 & 100 \\
\hline Total & 43 & 1 & 100 & & \\
\hline
\end{tabular}

Fuente: Los Autores

La tarea de investigar entre quienes no adquieren o abandonan el hábito de fumar y no se consideran enfermos y quienes persisten en el mismo convirtiéndose en enfermos de tabaquismo, dio como resultado lo siguiente:

Gráfico 8. Datos consolidados de estudiantes que fuman y han fumado antes y después de 15 años

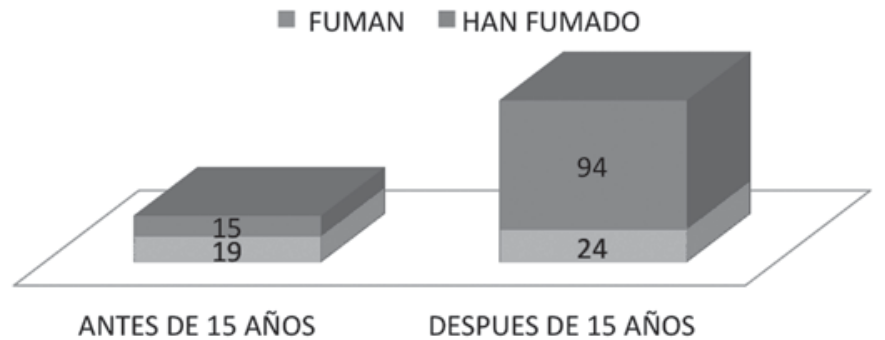

Fuente: Los Autores

Para ello solo utilizamos la base de datos de todos aquellos 152 que iniciaron el hábito de fumar y el momento de adquisición del hábito. La manera de saber cuántos y quienes abandonaron el hábito de fumar basto con restar a los 152 que lo iniciaron los 43 que aún fuman los cuales son: 109 que en la gráfica aparecen marcados con rojo y resulta de sumar (15 estudiantes que comenzaron a fumar antes de quince años + 94 que empezaron a fumar después de los 15 años.

Los que actualmente fuman 19 adquirieron el hábito antes de 15 años y 24 después de esa edad. De los 109 que no adquirieron el hábito o lo abandonaron 94 (86,24\%) probaron el cigarrillo después de los 15 años y solo 15 estudiantes, es decir solo el $13,76 \%$ de ellos aún fuma. Este hallazgo fue sometida a prueba estadística y nos proporciona un resultado sorprendente: Al realizar las pruebas cruzadas nos proporciona un OR de 4,96 (IC:2,2-11) p: 0,000051 lo que nos indica que quien adquiere el hábito o prueba el cigarrillo antes de 15 años tendrá 4,96 veces más probabilidad de ser fumador que quien lo hace después de 15 años. He considerado que este es tal vez el hallazgo más importante de la investigación.

Los resultados a la pregunta ¿Cuántos días al mes consumieron cigarrillos los fumadores y que cantidad de cigarrillos fueron consumidos? Se observan en la gráfica 9:

Gráfica 9. No de cigarillo consumidos al mes

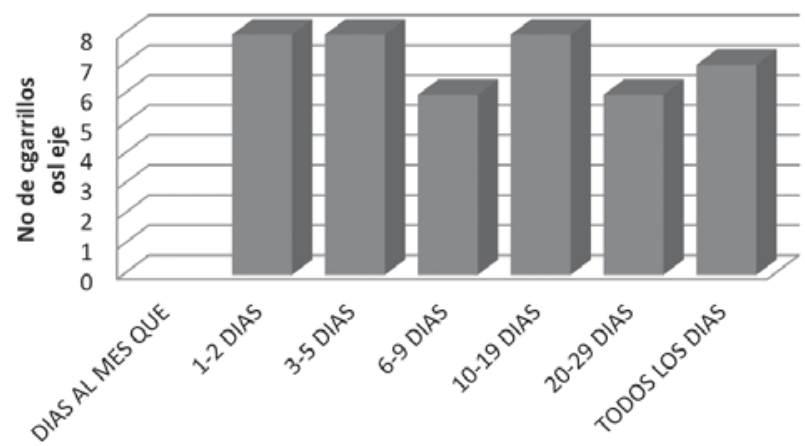

Fuente: Los autores

El resultado permite mostrar que el $19 \%$ solo fuman entre uno o dos días al mes que podría corresponder (F17 Trastornos mentales y del comportamiento debidos al consumo de tabaco) La información aquí proporcionada permite clasificar a los fumadores desde el fumador que prueba, experimentador, ocasional, habitual hasta el compulsivo.

La segunda pregunta: qué cantidad de cigarrillos consumen los fumadores por día? Obtuvo una respuesta que analizada conduce a afirmar que aproximadamente $10 \%$ de los fumadores son adictos

Los resultados muestran que el 59,68\% (aprox. 60\%) de los estudiantes de la universidad nunca han fumado y $40,32 \%$ afirma haber probado el cigarrillo al menos una vez en su vida. Este porcentaje es inferior al encontrado por la OMS en Bogotá en el año 2002 al 
aplicar la misma encuesta en adolescentes que arrojó un resultado de $62 \%$ de estudiantes que habían probado el cigarrillo al menos una vez en su vida.

\section{CONCLUSIONES}

La prevalencia de tabaquismo entre los estudiantes de la Universidad Autónoma del Caribe durante el 2013 es de 11,4\% encontrándose que es más baja que la prevalencia encontrada en otras universidades de la Costa Atlántica.

La prevalencia de esta enfermedad es el doble entre los hombres comparada con la prevalencia de las mujeres y en cuanto a jorna$\mathrm{da}$, en la diurna es mayor que la de aquellos que estudian en la nocturna siendo las diferencias estadísticamente significativas.

La mayoría de los encuestados iniciaron el hábito por curiosidad, siendo esta causa estadísticamente significativa.

Siendo un importante resultado, el hecho que aunque la mayoría de los encuestados iniciaron la prueba después de 15 años, sin embargo quienes lo hicieron por debajo de los 15 años, se encontró al compararlos, una diferencia estadísticamente significativa con relación a continuar el hábito cuando lo iniciaron por debajo de dicha edad; y coincidentes con las realizadas en otros estudios a nivel internacional.

Se hacen necesarias campañas educativas en los colegios que se inicie con los grupos de edades entre procedentes del estrato II y III fuman menos que los de los estratos ubicados en los extremos, tal vez por la presencia de la figura materna en casa y en canto a sitios donde más fuman son la casa y la Universidad.

\section{REFERENCIAS}

Adriani W, Spijker S, Deroche-Gamonet V, Laviola G, Le Moal M, Smit AB, Piazza PV. Evidence for enhanced neurobehavioral vulnerability to nicotine during periadolescence in rats. Journal of Neuroscience 2003, 23(11):4712-6.

American Cáncer Society. (2014) El humo de segunda mano. Cancer Facts \& Figures . Atlanta. Recuperado de: http:// www.cancer.org/espanol/cancer/ queesloquecausaelcancer tabacoycancer/humo-de-segunda-mano

Ariza C, Nebot M. (2002). Predictores de la iniciación al consumo de tabaco en escolares de enseñanza secundaria en Barcelona y Lleida. Rev Esp Salud Pública, 76: 227-38

Bello S., MIchalland H. S., Soto. S., Contreras. M., Salinas C., J. (2005). Efectos de la exposición al humo de tabaco ambiental en no fumadores. Revista chilena de enfermedades respiratorias, 21(3), 179-192. Recuperado de http://www.scielo.cl/scielo. php?script=sci_arttext\&pid=S0717-73482005000300005\&ln $\mathrm{g}=$ es\&tlng=es. 10.4067/S0717-73482005000300005

Belluzzi JD, Lee AG, Oliff HS, Leslie FM. (2003) Age-dependent effects of nicotine on locomotor activity and condi $\neg$ tioned place preference in rats. Psychopharmacology 2004, 174(3):389-95.
Boffetta P Et Al. (2005) Smockeless Tobacco use and Risck Of Cancer Of The Pancreas And Other Organs, Internacional Journal Of Cancer, 114(6): 992-995. Recuperado de www. ncbi.nlm.nih.gov/pubmed/15645430

Brody AL. (2006). Functional brain imaging of tobacco use and dependence. Journal of Psychiatric Research, 40(5):404-18.

California Environmental Protection Agency.(2005) Proposed identification of environmental tobacco Smoke as a toxic air contaminant: executive Summary. Sacramento, California environmental protection Agency, Recuperado de www.arb. ca.gov/carbis/regact/ets2006/app3exe.pdf

Centers for Disease Control and Prevention. (2008). Cigarette smoking among high school students-United States, 1991-2007. Morbidity and Mortality Weekly Report 2008b;57(25):689-91

Colby SM, Tiffany ST. (2000), Shiffman S, Niaura RS. Are ado-lescent smokers dependent on nicotine: a review of the evidence. Drug and Alcohol Dependence 2000a; 59(Suppl 1):S83-S95

COLPRENSA (2013, 31 de mayo) Salud. Al año mueren 21 personas a causa del tabaco. Citado por La Tarde.Com. Recuperado de http://www.latarde.com/entretenimiento/ salud/115406-alano-mueren-en-colombia-21000-personas-a-causa-del-tabaco

Congreso de Colombia. (2009). Ley 1335 de 2009. Articulo 19 numeral b Recuperado de: www.secretariasenado.gov.co/ senado/.../ley/2009/ley_1335_2009ht

DiFranza JR, Savageau JA, Fletcher K, Pbert L, O'Loughlin J, McNeill AD, Ockene JK, Friedman K, Hazelton J, Wood C, Dussault G, Wellman RJ.( 2007). Susceptibility to nicotine dependence: the Development and Assessment of Nicotine Dependence in Youth 2 study. Pediatrics 120(4):e974-e983

González C. (1999). Factores biopsicosociales que inciden en el consumo de tabaco en población infanto-juvenil. Bases para una educación preventiva. Sevilla: Consejería de Asuntos Sociales Comisionado para la Droga. Junta de Andalucía

Hughes JR. (2001) Distinguishing nicotine dependence from smoking: why it matters to tobacco control and psychi $\neg$ atry. Archives of General Psychiatry, 58(9):817-8.

Jiménez Ruiz C A, Solano Reina S, (2004) Tabaquismo Monografias Neumomadrid. Volumen VII

Johnston LD, O'Malley PM, Bachman JG, Schulenberg JE. (2007). Monitoring the Future: National Survey Results on Drug Use, 1975-2006. Volume I: Secondary School Students. Bethesda (MD): U.S. Department of Health and Human Services, National Institutes of Health, National Institute on Drug Abuse. NIH Publica $\neg$ tion No. 07-6205.

Levin ED, Rezvani AH, Montoya D, Rose JE, Swartzwelder HS. Adolescent-onset nicotine self-administration modeled in female rats. Psychopharmacology;169(2):141-9.

López A. D., Collishow H. E., Piha T. A., (1994). descriptive model of the cigarette epidemic in developed countries. Tobacco Control 3: 242-47.

Mathers CD, Loncar D,( 2002). Projection of global Mortalily and burden of disease from to 2030. Plos Medicine, 2006, 3 (11):4432. Recuperado de www.plosmedicine.org/.../journal. pmed.003044 
Ministerio de Salud de Argentina (2014) Programa Nacional del Control del tabaco. Recuperado de http://www.msal.gov.ar/ tabaco/index.php/informacion-para-profesionales/efectosdel-tabaco-en-la-salud

OMS. (2009). Informe epidemia mundial de tabaquismo, Recuperado de www.who.int/tobacco/global_report/2011/es/ index.html

(2013). Tabaco. Nota descriptiva 339 recuperado de http://www.who.int/mediacentre/factsheets/fs339/es/

OPS (2011) Informe sobre control de tabaco para la Región de las Américas. Recuperado de: http://www.paho.org/par/ index2.php?option=com_docman\&task=doc_view\&gid= 358\&Itemid $=239$

Organización Panamericana de la Salud, World Bank. (2000). La epidemia del tabaquismo: los gobiernos y los aspectos económicos del control del tabaco. Washington, DC:OPS: Publicación Científica 577

Panday S, Reddy SP, Ruiter RAC, Bergstrom E, DeVries H. (2007) Nicotine dependence and withdrawal symptoms among occasional smokers. Journal of Adolescent Health, 40(2):144-50.

Sánchez L. (2004). Tabaquismo en la infancia. Arch Bronconeumol. 40: 1-4.

Secretaría de Salud. (1998). Dirección General de Epidemiología/ SSA, México, D.F.

Slotkin TA. (2002)Nicotine and the adolescent brain: insights from an animal model. Neurotoxicology and Teratol $\neg$ ogy, 24(3):369-84.
Sociedad Americana Del Cáncer Y La Fundación Mundial Del Pulmón. (2013) El Atlas del Tabaco. Cuarta edición. Recuperado de http://www.tobaccoatlas.org/uploads/Files/ TA4-ChileLaunch-spanish.pdf

Tapia-Conyer R, Kuri-Morales P, Hoy-Gutiérrez MJ.(2001). Panorama epidemiológico del tabaquismo en México. Salud Publica México, 43:478-484.

Tomeo C. A., Field A. E., Berkey C. S., Golditz G.E., Frazier A. L.(1999). Weight concerns, weight control behaviors and smoking initiation. Pediatrics, 104: 918-24.

Torres OV, Tejeda HA, Natividad LA, O’Dell LE. (2008). Enhanced vulnerability to the rewarding effects of nicotine dur $\neg$ ing the adolescent period of development. Pharmacol-ogy, Biochemistry, and Behavior, 90(4):658-63.

Valdés, S. R., Michel J. M., Hernández L., Hernández M., Hernández M. (2002). Tendencias del consumo de tabaco entre alumnos de nuevo ingreso a la UNAM, 1989-1998. Salud Publica México 44 supl 1:S44-S53

Villalba J. R., Ariza. C. (2000). El tabaquismo en España: situación actual y perspectivas para el movimiento de prevención. Informe SESPAS. Barcelona: p. 3-15.

Waldran I, Lye D. (1990). Relatioships of teenage smoking to educational aspirationsand parent's education, J Subst Abuse, 2: 201-15

World Health Organization(1999) International Consultation on Tobacco and Youth: What in the world works? Final Conference Report.)Singapore. 